Revue des patrimoines

25 | 2014

Le patrimoine de la Grande Guerre

\title{
Création à Lyon des deux premières écoles françaises de blessés militaires en décembre 1914 : le cas de la maison forte de la Buire
}

\section{Catherine Guillot}

\section{(2) OpenEdition \\ Journals}

Electronic version

URL: http://journals.openedition.org/insitu/11343

DOI: 10.4000/insitu. 11343

ISSN: 1630-7305

Publisher

Ministère de la Culture

\section{Electronic reference}

Catherine Guillot, «Création à Lyon des deux premières écoles françaises de blessés militaires en décembre 1914 : le cas de la maison forte de la Buire », In Situ [Online], 25 | 2014, Online since 08 December 2014, connection on 25 June 2020. URL : http://journals.openedition.org/insitu/11343 ; DOI : https://doi.org/10.4000/insitu. 11343

This text was automatically generated on 25 June 2020.

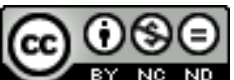

In Situ Revues des patrimoines est mis à disposition selon les termes de la licence Creative Commons Attribution - Pas d'Utilisation Commerciale - Pas de Modification 4.0 International. 
Création à Lyon des deux premières écoles françaises de blessés militaires en décembre 1914 : le cas de la maison forte de la Buire

Catherine Guillot

La maison forte de la Buire 
Figure 1

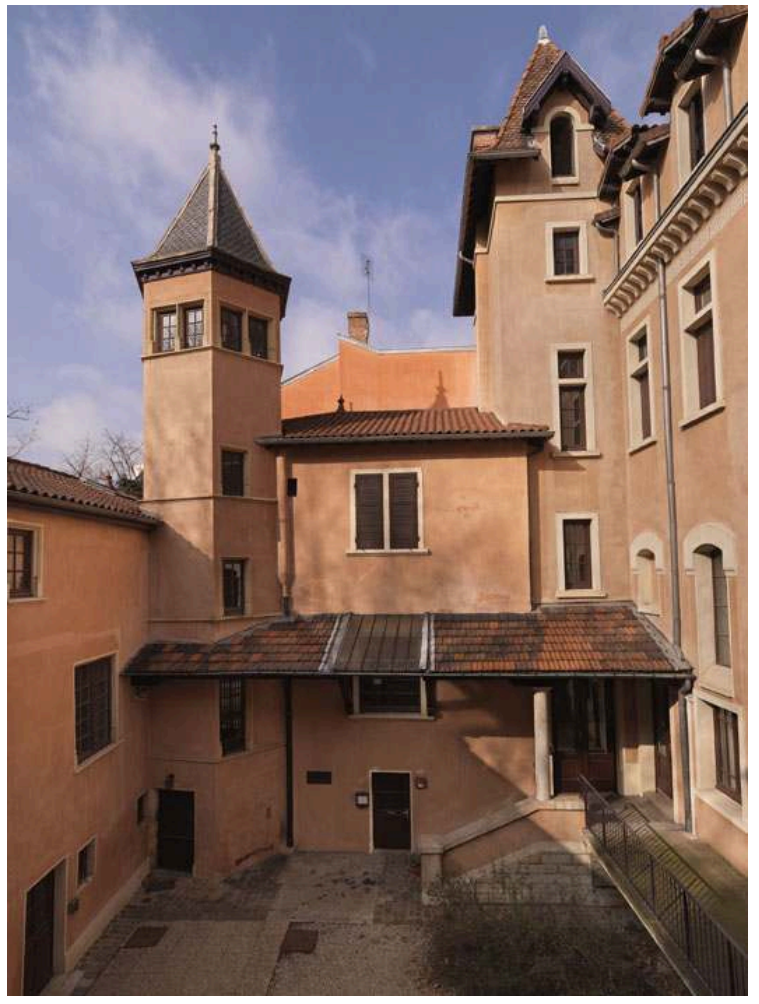

Vue de l'élévation nord, sur la cour.

Phot. Leroy, Thierry, 2013. @ Région Rhône-Alpes. Inventaire général du patrimoine culturel - ADAGP.

1 La maison forte de la Buire, établie sur la rive gauche du Rhône ${ }^{1}$, a été édifiée aux XIV et $\mathrm{XV}^{\mathrm{e}}$ siècles, au sein d'un vaste domaine rural, au sud de la route de Villeurbanne (fig. 1) ${ }^{2}$. Elle passe dans la famille Rachais en 1720 , qui en demeure détentrice jusqu'en $1814^{3}$. Celle-ci remanie fortement la maison, en particulier en aménageant vers 1774 le perron côté jardin ${ }^{4}$ (fig. 2). 
Figure 2

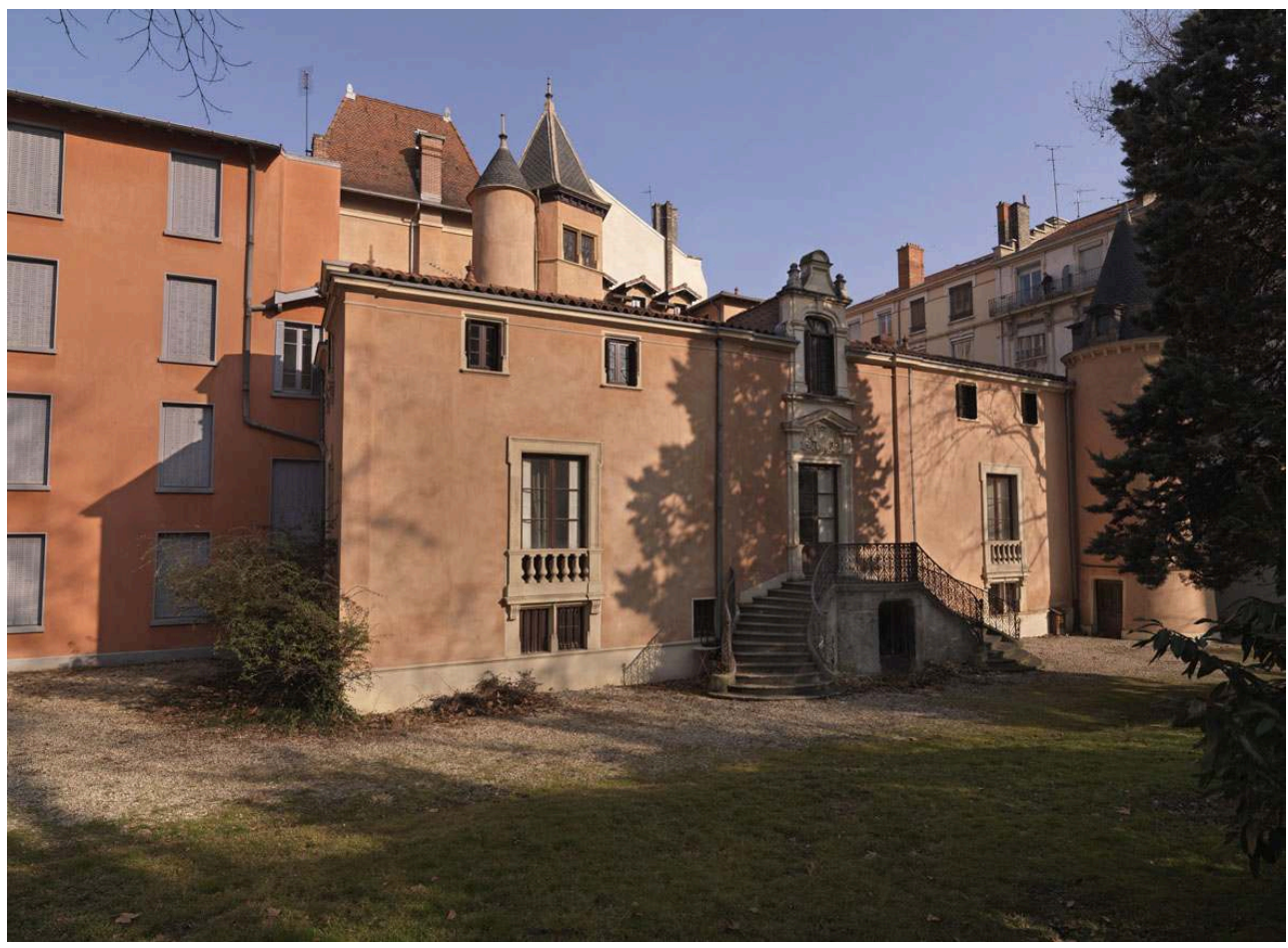

Vue de l'élévation est, sur le jardin.

Phot. Leroy, Thierry, 2013. @ Région Rhône-Alpes. Inventaire général du patrimoine culturel - ADAGP.

2 Madame Champanet, propriétaire de la maison dans le dernier quart du XIX siècle, la cède en 1885 à la toute jeune communauté des Petites Sœurs de l'Assomption, fondée en $1865^{5}$. Celle-ci se consacre à l'apostolat social auprès des milieux défavorisés, en particulier aux soins gratuits à domicile des malades nécessiteux. En 1891, elle fait construire perpendiculairement à la rue Rachais une chapelle, surmontée de dortoirs (fig. 3). 
Figure 3

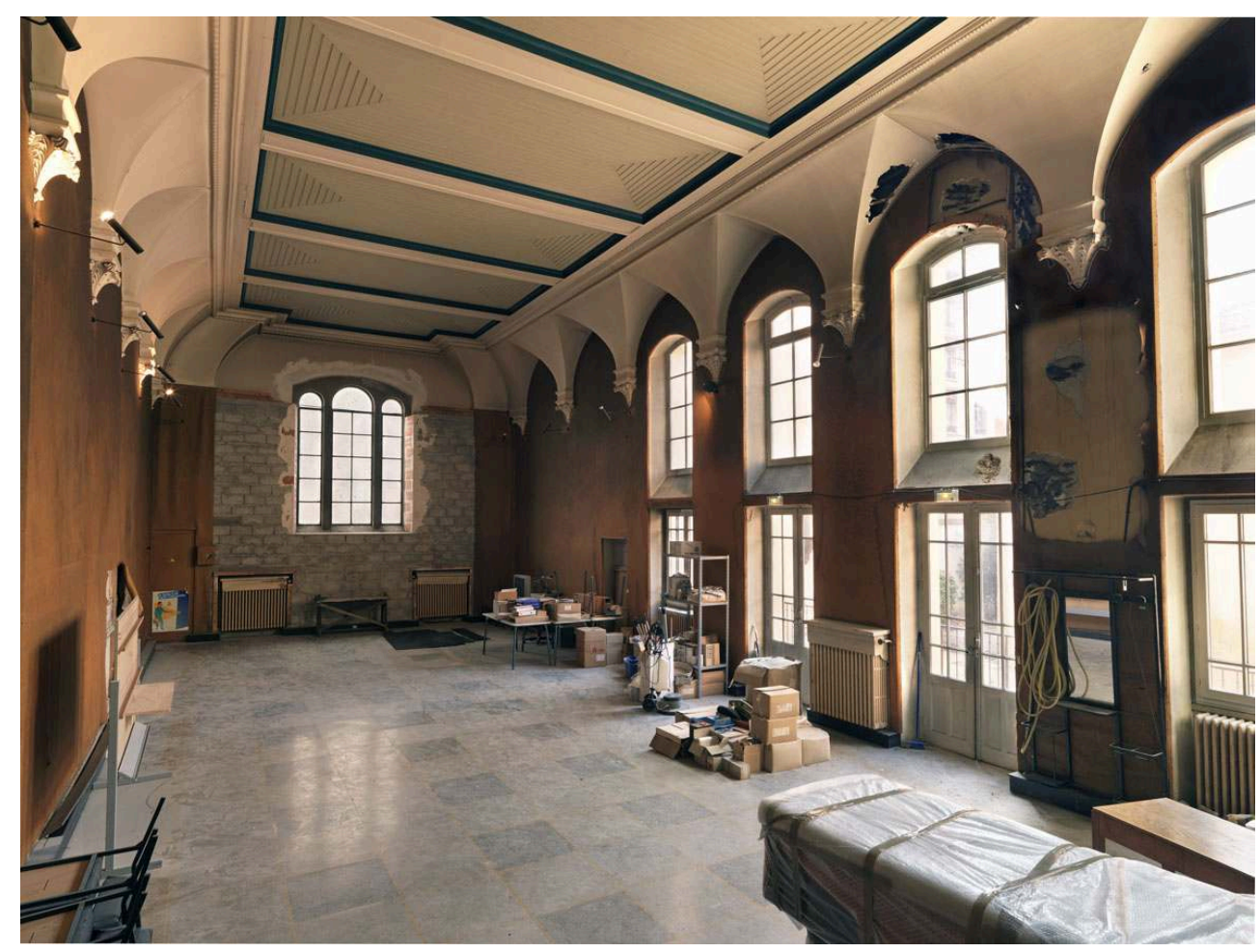

Vue intérieure de l'ancienne chapelle (état 2013).

Phot. Leroy, Thierry, 2013. @ Région Rhône-Alpes. Inventaire général du patrimoine culturel - ADAGP.

Sans doute suite à l'interdiction des Assomptionnistes en $1900^{6}$, dont les publications antidreyfusardes et antisémites sont dénoncées par les Républicains, les biens de la congrégation sont liquidés par l'administration des domaines, qui y installe le 8 novembre 1911 un gardien. Le 9 août 1913, l'immeuble est mis en vente et acquis par la Ville de Lyon' ${ }^{7}$. Les bâtiments sont transformés en foyer en juin 1914, à l'attention des jeunes filles travaillant à l'Exposition internationale qui se tient alors à Lyon. Mais la guerre remet en cause cette destination; ce n'est qu'en 1919 que les étudiantes pourront réinvestir les lieux.

\section{Le rôle d'Herriot}

En effet, sur le modèle d'une école visitée à Charleroi (Belgique), le maire de Lyon, Édouard Herriot, crée en décembre 1914 les deux premières écoles françaises de blessés militaires, l'une dans cette ancienne maison forte et l'autre (aujourd'hui détruite) dans l'hôpital auxiliaire nº2 202 bis, situé 25 chemin de Tourvielle au Point-du-Jour.

5 Dès le début de la guerre, Herriot prend la mesure de l'ampleur du conflit, des mutilations infligées aux soldats et envisage un après-guerre dans lequel ces mutilés pourront retrouver une place dans la société française. Il comprend que les hommes ne pourront retourner au travail qu'ils effectuaient avant le conflit, en raison de leurs mutilations et des changements induits par la guerre. Il veut également éviter d'isoler les blessés dans de grands hôpitaux. Il s'inspire de la doctrine du colonel Charles Ardant du Picq (1821-1870), qui, dans son ouvrage posthume Études sur le combat ${ }^{8}$, met en avant la psychologie humaine et la solidarité, et non plus seulement l'effet de masse dans les 
combats. Herriot souhaite en particulier «rendre à l'amputé de guerre sa triple autonomie professionnelle, intellectuelle et morale ", en le rendant maître d'un travail'9. Il assure lui-même la publicité de son œuvre par des conférences, comme celle prononcée à Saint-Étienne, publiée dès 1915. En octobre et décembre de la même année, des photographies officielles des mutilés en apprentissage sont également réalisées (fig. 4).

Figure 4

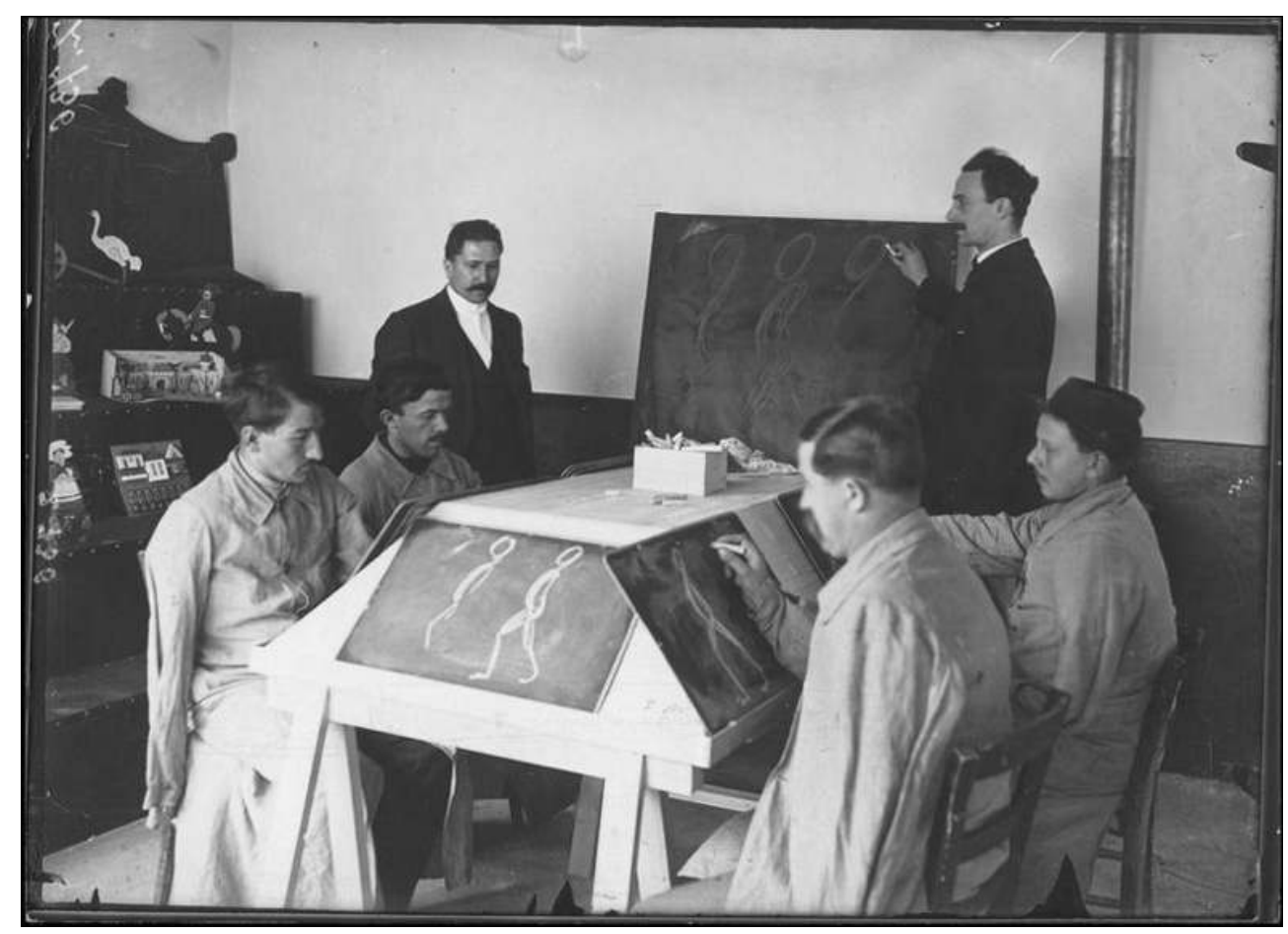

Cours de dessin et exemples de jouets réalisés à l'arrière-plan, octobre 1915.

Phot. Opérateur Z (Référence APZ0000736A). @ Ministère de la Culture, médiathèque de l'architecture et du patrimoine.

\section{Une nouvelle destination, des formations adaptées}

6 Les locaux de l'ancienne maison forte, rue Rachais, sont ainsi aménagés progressivement et les formations se mettent également en place petit à petit. Une bibliothèque est créée dans l'ancien logis et des dortoirs dans la chapelle. L'hôpital Desgenettes fournit aux anciens soldats les pilons, les jambes en bois, les lunettes afin qu'ils retrouvent une certaine autonomie physique.

7 Une première idée est de faire réaliser par les blessés des jouets en bois car ceux-ci provenaient avant le déclenchement du conflit principalement d'Allemagne, de Nuremberg en particulier (fig. 5). Des figurines patriotiques sont notamment conçues, dont des Alsaciennes en costume. Jusqu'en 1915, le façonnage du bois est exécuté chez le fournisseur qui a le matériel adéquat et le travail de finition des jouets effectué par les élèves de l'école. La guerre se prolongeant, il est décidé d'acheter du matériel pour que toute la production se fasse sur place. 
Figure 5

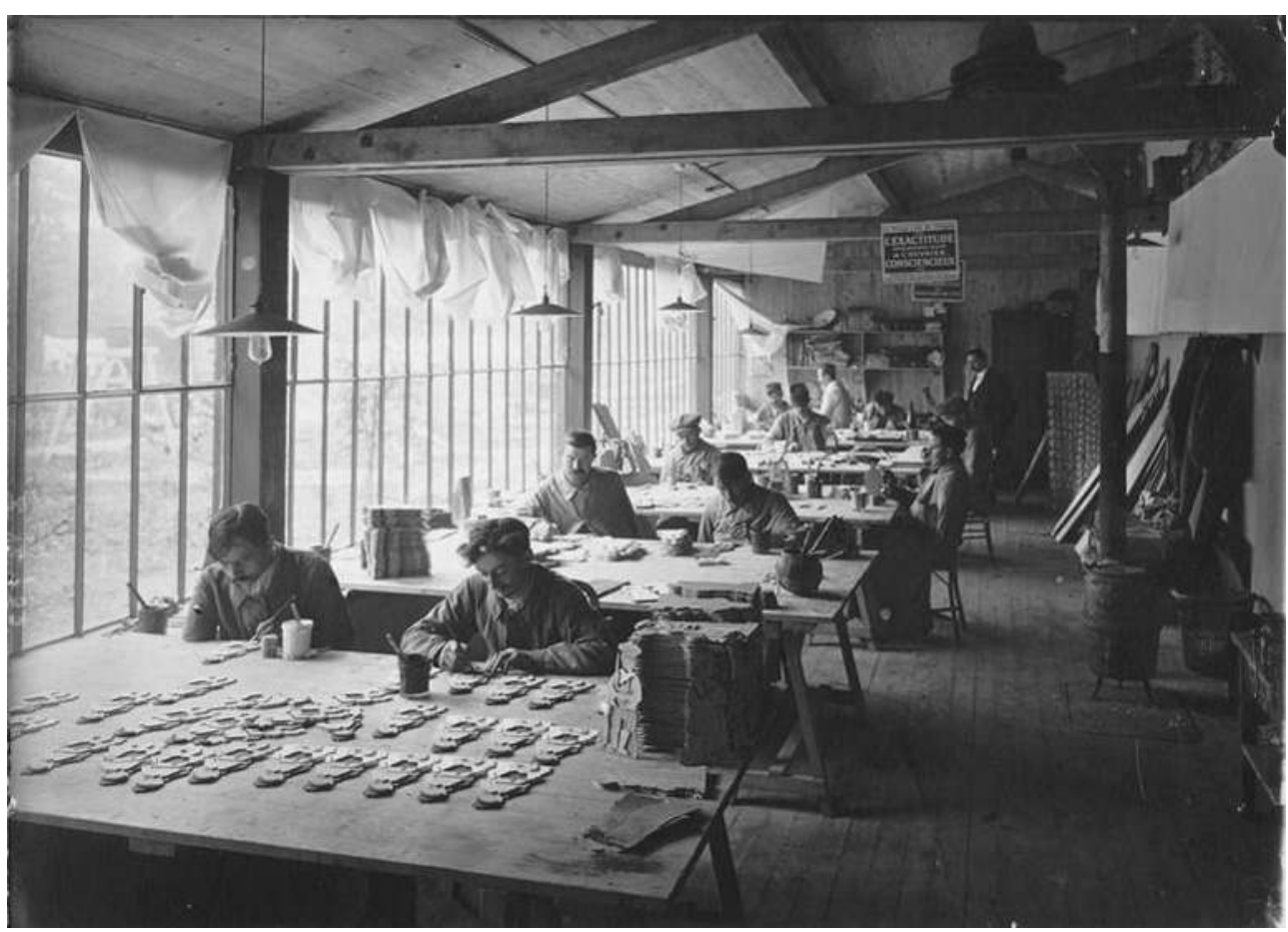

La peinture des jouets dans l'atelier, en 1915.

Phot. Opérateur Z (Référence APZ0000724A). (c) Ministère de la Culture, médiathèque de l'architecture et du patrimoine.

8 En 1915, Joffre accepte de donner son nom à l'école de la rue Rachais et celle du chemin de Tourvielle devient l'école Foch. Le vainqueur de la Marne est choisi par la Ville en raison de ses succès et de l'affection qu'il voue, selon Herriot, aux soldats.

9 À partir de 1915 également, sont organisés des cours d'anglais et de comptabilité ; un potager est aménagé dans le jardin. Le peintre lyonnais Tony Tollet (1857-1953), deuxième Grand prix de Rome en 1885, vient donner des cours de dessin. En 1916, on compte rue Rachais, selon les mois, entre 12 et 25 externes et entre 89 et 92 mutilés internes ${ }^{10}$.

D'autres formations sont progressivement proposées. Au final, à l'école Joffre sont organisés les cours de comptabilité, de papeterie-reliure (fig. 6), de fabrication des jouets, de passementerie et perles, tandis que l'école Foch accueille les formations de cordonnier, galochier, tailleur d'habits, menuisier ébéniste, fourreur, orthopédiste, opérateur radiotélégraphiste et horticulteur. 
Figure 6

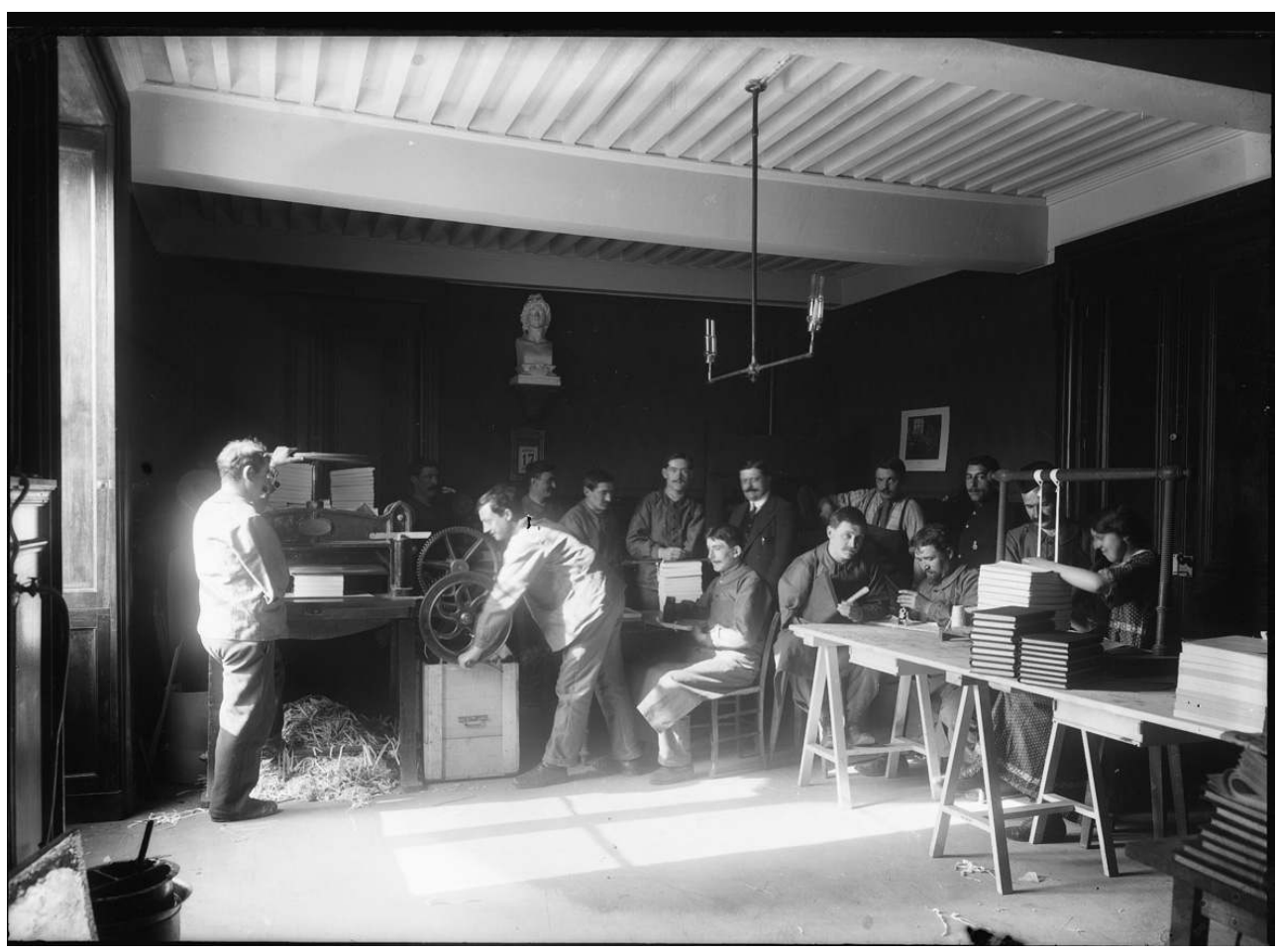

Une des pièces abritant l'atelier des relieurs-brocheurs, vers 1915.

Phot. Inconnu, fonds Jules Sylvestre, P0546_S 1289. (c) Bibliothèque municipale de Lyon.

11 Les élèves sont rigoureusement sélectionnés sur leur moralité et leur sérieux. Des panneaux sont accrochés dans les ateliers, rappelant à l'apprenti ses devoirs d'exactitude et de conscience professionnelle. Les écarts (manque d'assiduité, absences prolongées, ivrognerie) sont sanctionnés, jusqu'à l'exclusion. Les archives ne permettent cependant pas de suivre le nombre anticipé de départs, volontaires ou non.

La durée moyenne de l'apprentissage varie de 6 à 18 mois selon les métiers : 6 mois pour la passementerie et les perles, 8 mois pour la comptabilité, les radiotélégraphistes et les galocheurs, un an pour la papeterie-reliure, les cordonniers, les fourreurs et les horticulteurs, enfin, 18 mois pour la fabrication des jouets, les tailleurs d'habits, les menuisiers ébénistes et les orthopédistes. Sa formation achevée, le mutilé reçoit un certificat d'aptitude professionnelle (diplôme créé en 1911 et appelé CAP à partir de 1919).

13 Beaucoup d'hommes doivent abandonner complètement leur premier métier; dans une France encore extrêmement rurale, nombreux sont les cultivateurs qui ne peuvent plus exercer les travaux des champs. Le degré et la nature des mutilations sont évidemment primordiaux dans les possibilités de reconversion des anciens soldats. Les mutilés des jambes sont en particulier les plus difficiles à faire embaucher (fig. 7) : ils ne peuvent être coursiers, ni emprunter facilement un escalier dans une boutique, alors qu'à Lyon les magasins situés dans les immeubles récents ont systématiquement leurs réserves installées dans un demi-étage au-dessus du magasin. 
Figure 7

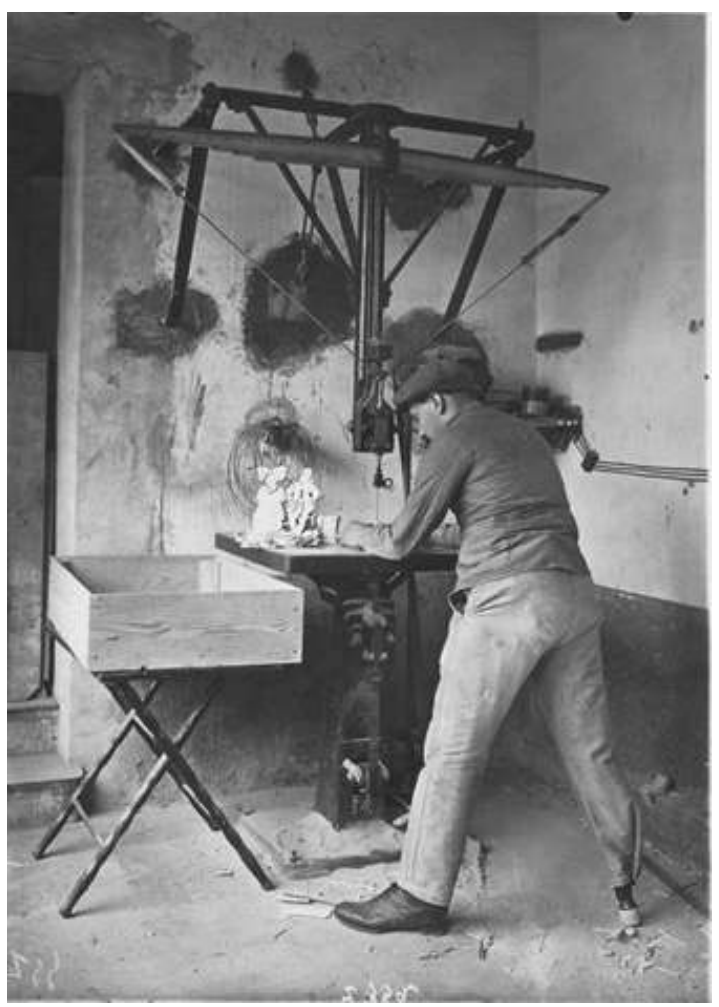

Un scieur-découpeur unijambiste à l'atelier de fabrication des jouets, en décembre 1915.

Phot. Opérateur Z (Référence APZ0000732A). @ Ministère de la Culture, médiathèque de l'architecture et du patrimoine.

\section{La Société française du jouet}

L'école Foch ferme en $1919^{11}$. Son action est prolongée par la Société française du jouet, créée le 30 octobre 1919, qui maintient une activité de fabrication rue Rachais. Il s'agit d'une œuvre à caractère philanthropique, destinée à fournir du travail aux invalides de guerre, et susceptible de leur reverser des dividendes. La Ville, qui a consenti un bail de six ans, en est actionnaire et prête le matériel de fabrication. L'entreprise prend la forme d'une société anonyme fondée par Louis Lumière dont le but est la confection et la vente de jouets en bois fabriqués par les invalides de guerre ${ }^{12}$. Elle n'est plus installée dans la maison forte mais dans une partie des ateliers construits sur le terrain de la propriété désormais scindé par un mur de clôture. La maison forte peut à nouveau accueillir de jeunes étudiantes ; le foyer est désormais géré par l'Université.

L'économie reprend ses droits et le caractère philanthropique de l'entreprise s'estompe. Une lettre datée de 1920 d'un administrateur du Jouet français, Lamarche, éclaire d'un tout autre jour le fonctionnement de l'atelier. Il s'agit d'un véritable réquisitoire contre les mutilés : ceux-ci ont mauvais esprit, ne travaillent pas comme le feraient des travailleurs valides, mais veulent néanmoins des salaires toujours plus hauts et parlent d'exploitation patronale. Les mutilés se jalousent, sont trop prétentieux en matière de salaires, manifestent peu d'ardeur au travail, ignorent la solidarité et méconnaissent les excellentes intentions de la Ville de Lyon et les âmes généreuses qui s'intéressent à leur sort... En 1921, la Société du jouet n'a plus de 
commandes; elle est dissoute le 31 juillet 1922. Rachetée, elle devient la Manufacture lyonnaise de jouets et douze blessés de guerre y demeurent employés ${ }^{13}$.

Il ne semble pas exister de suivi des mutilés diplômés des écoles professionnelles de rééducation de la Ville de Lyon, qui permettrait ainsi de mesurer le succès de cette entreprise pionnière. Il serait également intéressant de retrouver des témoignages directs de mutilés ayant été formés à Lyon, seule la politique volontariste des autorités municipales et la perception (extrêmement négative) d'un des administrateurs nous étant connues.

La Seconde Guerre mondiale va rattraper l'ancienne maison forte de la Buire. En 1941, le recteur de l'académie de Lyon depuis 1931, André Lirondelle (1879-1952), est sanctionné par le régime de Vichy pour avoir refusé de publier une affiche de propagande auprès des étudiants lors d'une visite du maréchal Pétain. Il est déplacé à Bordeaux ${ }^{14}$. En février 1941, dans un geste symbolique de résistance, la Ville, toujours propriétaire des lieux, et l'Université décident de donner à la maison le nom d'André Lirondelle ${ }^{15}$. Aujourd'hui, rien ne laisse deviner que la résidence universitaire Lirondelle a abrité durant cinq ans une des deux premières écoles professionnelles de soldats mutilés de la Première Guerre mondiale.

\section{NOTES}

1. - Voir le site : http://patrimoine.rhonealpes.fr/dossier/maison-forte-dite-chateau-de-la-buirecouvent-de-petites-soeurs-de-l-assomption-ecole-de-reeducation-professionnelle-de-blessesmilitaires-de-la-ville-de-lyon-dite-ecole-joffre-maison-des-etudiantes-lirondelle/ c7c229ee-33af-4cd4-8427-253fba845945.

2. - Elle est aujourd'hui située au 6 de la rue Rachais.

3. - BAZIN, Georges. «Le Château de la Buire ». Rive Gauche, n¹3, p. 14-15.

4. - SALOMON, Émile. Les Châteaux historiques du Lyonnais et du Beaujolais. Lyon: édition de la « République lyonnaise », 1938, tome II, p. 7-8.

5. - BAZIN, op. cit., p. 15.

6. - Voir le site : http://kadoc.kuleuven.be/relins/relins/scherm12_1.html.

7. - Arch. mun. Lyon, 726 WP 134.

8. - ARDANT DU PICQ, Charles. Études sur le Combat. Paris : Hachette et Dumaine, 1880. En ligne sur Gallica : http://gallica.bnf.fr/ark:/12148/bpt6k864841/f6.image.

9. - Société d'études économiques du département de la Loire. L'assistance aux Mutilés de la Guerre. Conférence de M. É. Herriot. Saint-Étienne : librairie Chevalier, 1915.

10. - Arch. mun. Lyon, 112 WP 043.

11. - Arch. mun. Lyon, 726 WP 135 et 112 WP 044.

12. - Arch. mun. Lyon, 726 WP 135.

13. - Arch. mun. Lyon, 726 WP 135.

14. - MAZON, André. "André Lirondelle (1879-1952)». Revue des études slaves, tome 29, fascicule 1-4, 1952, p. 108-112, en ligne: http://www.persee.fr/web/revues/home/prescript/article/ slave_0080-2557_1952_num1_1584.

15. - Arch. mun. Lyon, 726 WP 134. 


\section{ABSTRACTS}

In 2013, during the preparation of a heritage inventory file on a former fortified house known as the Buire castle, located in the third arrondissement of Lyon, the story came to light of the first two schools specially created in France for the war wounded. With the support of his municipal council, the mayor of Lyon, Edouard Herriot (1872-1957), decided in December 1914 to create two special professional schools for mutilated soldiers. One of these schools (Joffre) was set up in this former fortified house, whilst the other (Foch) was set up in an auxiliary hospital, which has since disappeared. The article looks in particular detail at the Joffre school, where some of the buildings are still in existence.

Au détour d'un dossier d'Inventaire réalisé en 2013 à l'occasion de travaux d'aménagement dans un foyer d'étudiantes situé dans le $3^{\mathrm{e}}$ arrondissement de Lyon, dans l'ancienne maison forte de la Buire, a ressurgi l'histoire des deux premières écoles de blessés militaires en France créées durant la Première Guerre mondiale. En effet, le maire de Lyon, Édouard Herriot (1872-1957), et son conseil municipal établissent dès décembre 1914 deux écoles professionnelles destinées aux soldats mutilés, l'une dans cette ancienne maison forte (école Joffre) et l'autre (aujourd'hui détruite) dans un hôpital auxiliaire (école Foch). Cet article met plus particulièrement l'accent sur l'école Joffre, dont les bâtiments sont partiellement conservés.

\section{INDEX}

Keywords: First World War, Lyon, mutilated soldiers, Buire, professional school, Herriot, Tourvielle, Ardant du Picq, Joffre school

Mots-clés: Première Guerre mondiale, Lyon, mutilés, Buire, école professionnelle, Herriot, Tourvielle, Ardant du Picq, école Joffre

\section{AUTHOR}

\section{CATHERINE GUILLOT}

Conservatrice en chef du patrimoine, conservation régionale des monuments historiques, DRAC Rhône-Alpes catherine.guillot@culture.gouv.fr 